\title{
ERRATUM
}

\section{Erratum to: Delineating the respective impacts of stochastic curl- and grad-forces in a family of idealized core genetic commitment circuits}

\author{
Marc Turcotte $e^{1,2, *}$ \\ ${ }^{1}$ Department of Mathematics, The University of Texas at Arlington, Arlington, TX 76109, USA \\ 2 Biological Sciences, The University of Texas at Dallas, Richardson, TX 75080, USA \\ * Correspondence: marc.turcotte@utdallas.edu, marc.turcotte@uta.edu
}

\section{Erratum to: Quant Biol}

DOI 10.1007/s40484-016-0070-5

The original version of this article contained an error in panel D of Figure 4. The original panel D erroneously duplicated panel C. The author apologize for this oversight. The corrected Figure 4 can be found below. 
A

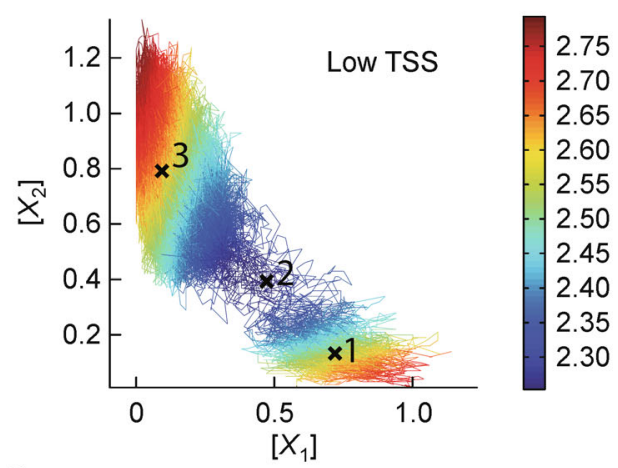

C

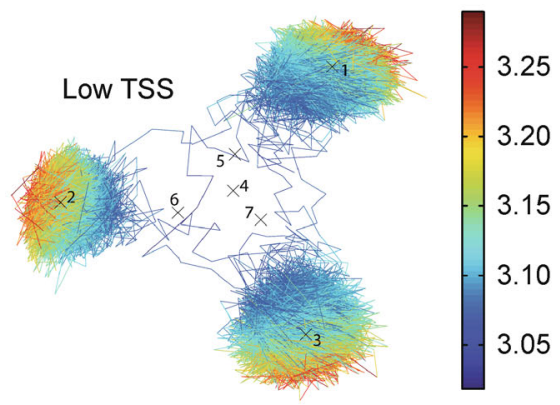

$\mathrm{E}$

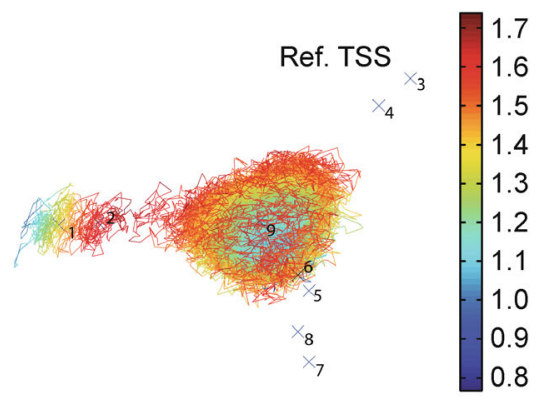

G

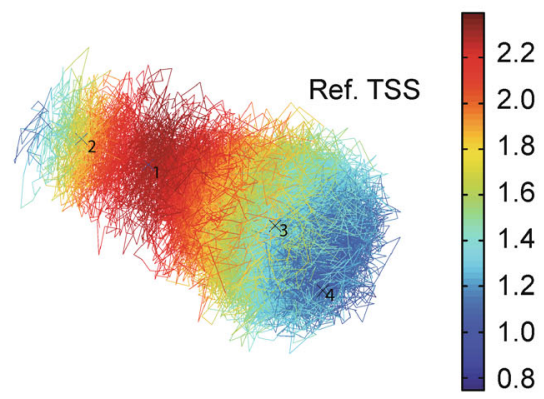

B

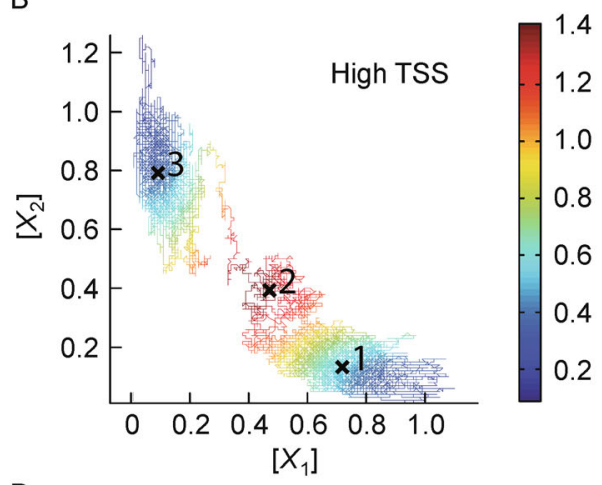

D

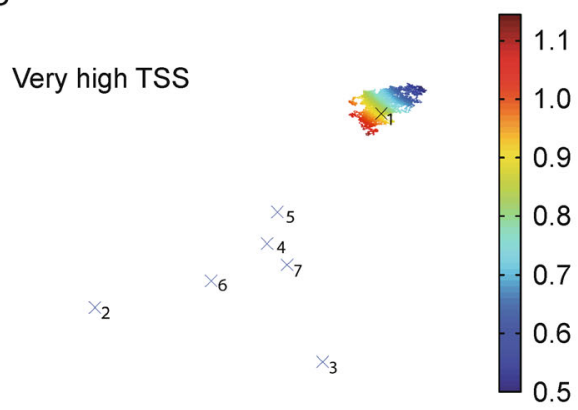

$\mathrm{F}$

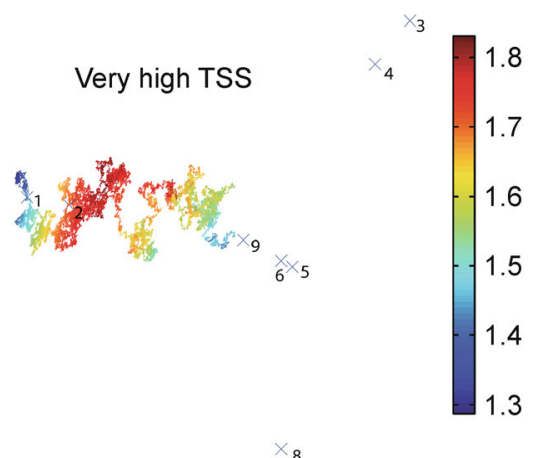

$\mathrm{H}$

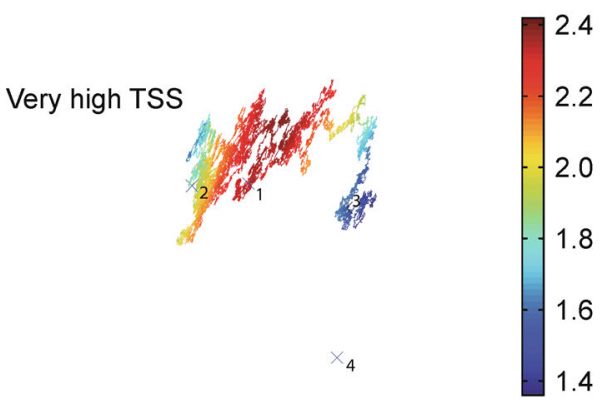

Figure 4. Diagrams of curl sampled along one single stochastic trajectory. Propagation is displayed by sequential colored line segments joining successive locations along the stochastic track. Only a relevant part of the track is displayed; details in the text. The contribution by components 1, 2 (top row) and 1, 2, 3 (rows 2, 3 and 4) to the local curl modulus is indicated by the color ranging from each figure's minimum curl to its maximum. The curl scale is linear. Rows 1, 2, 3 and 4 are for DS1, DS2, DS3 and DS4 respectively. Left and right columns are for low TSS and high TSS respectively. In high TSS, the messenger RNA sub-manifold is essentially at rest. The $X_{1}, X_{2}$ and $X_{3}$ axes are omitted on panel $\mathrm{C}$ through $\mathrm{H}$ because they would force an adversely distant viewpoint making details of the tracks imperceptible. See Table S1. 